\title{
Thermal Rectification in Billiardlike Systems
}

\author{
Jean-Pierre Eckmann ${ }^{1,2}$ and Carlos Mejía-Monasterio ${ }^{1}$ \\ ${ }^{1}$ Département de Physique Théorique, Université de Genève, Switzerland \\ ${ }^{2}$ Section de Mathématiques, Université de Genève, Switzerland
}

(Received 29 May 2006; published 28 August 2006)

\begin{abstract}
We study the thermal rectification phenomenon in billiard systems with interacting particles. This interaction induces a local dynamical response of the billiard to an external thermodynamic gradient. To explain this dynamical effect we study the steady state of an asymmetric billiard in terms of the particle and energy reflection coefficients. This allows us to obtain expressions for the region in parameter space where large thermal rectifications are expected. Our results are confirmed by extensive numerical simulations.
\end{abstract}

DOI: 10.1103/PhysRevLett.97.094301

Introduction. - The emergence of macroscopic behavior from the underlying microscopic dynamical laws is a major puzzle of theoretical physics. However, despite its long history, attempts to link these macroscopic laws to the underlying microscopic dynamics have not been conclusive thus far $[1,2]$.

With the advent of nanotechnology, the study of dynamics of systems at a nanoscopic scale starts to play a central role. In view of the broad spectrum of possible applications, the investigation on how the properties of the microscopic dynamics can be used to understand and thus to control the behavior of nanodevices has attracted much attention [3].

One such example is the phenomenon of thermal rectification (TR). A thermal rectifier is a device in which the magnitude of the heat current depends on the sign of the imposed temperature gradient. In the past there have been several experimental attempts to design such a device (see, e.g., [4], and references therein). However, a possible theoretical mechanism to explain the phenomenon from first principles has been proposed only recently [5].

So far, there is no unique framework to explain the phenomenon of thermal rectification. In [5] and in later papers [6-8], different models of anharmonic oscillators coupled to stochastic heat baths have been studied. Because of anharmonic terms, the width and position of an "effective" phonon band depend on temperature. It has been shown that by tuning the anharmonicities of two or more different coupled chains it is possible to control the effective overlap between the phonon bands of the two chains. In particular, the extent of this overlap can be made to depend on the sign of the imposed thermal gradient, thus leading to TR. A further step to devise a thermal transistor has been discussed in [7] in terms of the negative differential thermal resistance observed in some anharmonic chains. More recently, in [9] a thermal rectifier based on a spin-boson nanojunction model has been discussed.

In this Letter we study the phenomenon of thermal rectification in low dimensional "billiard" Hamiltonian systems. Roughly speaking, a billiard consists of an ensemble of free particles colliding elastically with a fixed
PACS numbers: 44.10.+i, 05.45.-a, 05.70.Ln

boundary. Furthermore, to study heat transport and thus rectification, we consider that the billiard is coupled at two different openings of its boundary with two different thermodynamic baths.

In a billiard, as described above, TR is identically zero irrespective of the geometry of the boundary, as the strengths of the fluxes inside the billiard are determined only by its coupling with the external baths. Here, we show that if the particles are allowed to interact (as defined below), then rectification of the heat flux is possible and can be quite large.

The model we consider consists of a gas of noninteracting point particles of mass $m$ that move freely inside a onedimensional open channel made of 2 identical twodimensional cells (like chaotic billiards), connected through openings of size $\delta$. At the center of each cell, there is a fixed, freely rotating disc of radius $R$ with which particles interact, exchanging energy as follows: when a particle hits a disc, it exchanges its tangential velocity $v_{t}$ with the angular velocity $\omega$ of the disc, while the normal component $v_{n}$ is reflected [10]:

$$
\omega^{\prime}=v_{t}, \quad v_{t}^{\prime}=\omega, \quad v_{n}^{\prime}=-v_{n} .
$$

This model was introduced in [10,11] (with a different geometry) to study macroscopic transport in terms of its microscopic dynamics. It was found that the disc mediated an effective interaction among the particles that leads to a realistic macroscopic transport behavior. Moreover, at each cell this effective interaction is sufficient for the establishment of approximate local thermal equilibrium $[10,12]$.

As a result of the interaction, when a particle enters the billiard from one of the reservoirs, its probability to reach the other reservoir, i.e., the transmission coefficient, depends not only on the geometry of the billiard but also on the local thermodynamic gradients. This dynamical effect was studied in detail in [13] in a billiard of (effectively) interacting particles with a geometry similar to the one that we describe below (see also [12]).

To set up the thermal rectifier, we exploit the dependence of the dynamical effect described above on the geometry of the cell. We choose the size of the disc in 
the left cell $(-)$ smaller than the disc in the right cell $(+)$. The transmission coefficient will then depend differently on the thermodynamic fields, and this leads to TR. Similar ideas have been discussed in [8] for dissimilar anharmonic lattices in terms of the thermal resistance at the interface. The geometry of our setup is described in Fig. 1.

To impose nonequilibrium, the two cells are connected at the ends to two reservoirs of particles through openings of the same size $\delta$. The reservoirs are idealized as infinite chambers containing an ideal gas at a certain density $n$ and temperature $T$ [11].

From the left reservoir, particles are injected into the $(-)$ cell at a constant rate $j_{>}$with Boltzmann distributed energy with a mean temperature $T_{>}$. Analogously, the right reservoir injects particles into the $(+)$ cell at rate $j_{<}$and temperature $T_{<}$. The rate at which energy is injected into the system from the left and right reservoirs is then given by $q_{>}=\frac{3}{2} j_{>} T_{>}$and $q_{<}=\frac{3}{2} j_{<} T_{<}$, respectively [12]. Each injected particle is eventually reabsorbed by one of the reservoirs, and this happens when it hits that reservoir.

The density $n$ and temperature $T$ of the reservoir are related with its injection rates as $n \propto j / T^{1 / 2} \propto j^{3 / 2} / q^{1 / 2}$. At the junction between the two cells, particles cross from $(-)$ to $(+)$ at a rate $j_{R}$ and from (+) to (-) at a rate $j_{L}$. We denote by $q_{R}$ and $q_{L}$ the corresponding energy rates.

In the steady state the conservation of particle number and energy can be expressed as a set of balance equations [13],

$$
\begin{aligned}
j_{L} & =\frac{\alpha_{L}^{+}\left(1-\alpha_{L}^{-}\right) j_{>}+\left(1-\alpha_{R}^{+}\right) j_{<}}{1-\alpha_{L}^{+} \alpha_{R}^{-}}, \\
q_{L} & =\frac{\gamma_{L}^{+}\left(1-\gamma_{L}^{-}\right) q_{>}+\left(1-\gamma_{R}^{+}\right) q_{<}}{1-\gamma_{L}^{+} \gamma_{R}^{-}},
\end{aligned}
$$

where $\alpha_{L}^{-}$is the reflection probability for the particles that are injected into the cell (-) from the left and $\alpha_{R}^{-}$for the particles injected into the cell $(-)$ from the right and similarly for the $(+)$ cell. The parameters $\gamma$ stand for the

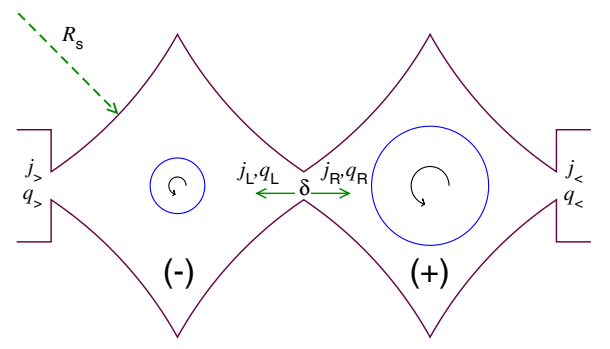

FIG. 1 (color online). Geometry of the rectifier. The boundary of each cell is made of four arcs of circles of radius $R_{s}$. The radius of the rotating discs is chosen to be larger than the width of the openings $\delta$, so that no particle can cross the cell without undergoing any collision. The arrows represent the flows of particles across the openings, and the symbols fix the notation for the rates of particles $j$ and energy $q$. Unless otherwise specified, the numerical results in this Letter correspond to a rectifier with discs of radius 0.04 for the $(-)$ cell and 0.18 for the (+) cell, $\delta=0.04$, and $R_{s}=1$. energy reflection coefficients with the same indexing as the $\alpha$ 's. Analogous equations are obtained for $j_{R}$ and $q_{R}$, by exchanging $L \leftrightarrow R,+\leftrightarrow-$, and $>\leftrightarrow<$.

Note that out of equilibrium the reflection from the left is not necessarily the same to the reflection from the right due to the effective particle interaction.

In terms of the rates at the junction the particle current is obtained by $\Phi_{n}=j_{R}-j_{L}$ and analogously, the heat current is $\Phi_{u}=q_{R}-q_{L}$. Using (2) the currents are

$$
\Phi_{n}=\frac{\tau_{L}^{\alpha} j_{>}-\tau_{R}^{\alpha} j_{<}}{1-\alpha_{L}^{+} \alpha_{R}^{-}}, \quad \Phi_{u}=\frac{\tau_{L}^{\gamma} q_{>}-\tau_{R}^{\gamma} q_{<}}{1-\gamma_{L}^{+} \gamma_{R}^{-}},
$$

where $\tau_{y}^{x}=\left(1-x_{y}^{-}\right)\left(1-x_{y}^{+}\right)$, with $x=\{\alpha, \gamma\}, \quad y=$ $\{L, R\}$, are the total transmission probabilities for the particles that enter the channel from the $y$ side. The currents in (3) are obtained as the difference of the products of these transmission probabilities with the injection rates, weighted by combinatorial factors that account for multiple reflections between $(-)$ and $(+)$.

Reflection coefficients for a single cell. - In [13] a phenomenological theory for the reflection probabilities $\alpha_{L_{R}}^{ \pm}$ was obtained. These results are for a single cell with injection of particles and energy at rates $j_{>}, q_{>}$from the left and $j_{<}, q_{<}$from the right. The reflection probabilities depend on the local thermodynamic fields, that is,

$$
\alpha_{L} \equiv \alpha_{L}\left(j_{>}, q_{>}, j_{<}, q_{<}\right), \quad \gamma_{R} \equiv \gamma_{L}\left(j_{>}, q_{>}, j_{<}, q_{<}\right) .
$$

Since the dynamics is homogeneous in energy, the number of independent variables in (4) is reduced to three. It was found convenient to distinguish two contributions: one (called $\alpha_{G}$ and $\gamma_{G}$ ) of purely geometrical origin, and another (called $\varepsilon$ and $\nu$ ) of dynamical origin arising as the system is driven out of equilibrium:

$$
\begin{gathered}
\alpha_{L}=\alpha_{G}(n)+\varepsilon_{L}(n, \delta j, \delta q), \\
\gamma_{R}=\gamma_{G}(n)+\nu_{L}(n, \delta j, \delta q),
\end{gathered}
$$

where $\delta j=\left(j_{>}-j_{<}\right) /\left(j_{>}+j_{<}\right)$and similarly for $\delta q$. By definition, $\varepsilon$ must vanish at equilibrium, i.e., $\varepsilon_{R}(n, 0,0)=$ 0 , and by left-right symmetry of the single cell, $\varepsilon_{L}=-\varepsilon_{R}$.

Detailed analysis showed the following: (i) At equilibrium $\alpha_{L}=\alpha_{G}(n)$. (ii) $\alpha_{G}(n)$ saturates, for low and high densities, to two constant values connected by a linear dependence for intermediate densities. (iii) $\varepsilon$ vanishes on a submanifold of $\left(j_{>}, q_{>}, j_{<}, q_{<}\right)$where $T_{>}=T_{<}$. This means that deviations from the equilibrium $\alpha$ 's occur for only $\delta j \neq 0$. (iv) Close to equilibrium, the dynamic component depends linearly on the local gradients of the particle injection, namely, $\varepsilon_{R}= \pm \mathcal{A} \delta j, \quad \nu_{L}= \pm \mathcal{B} \delta j$. Note that $\nu$ depends on $\delta j$, not on $\delta q$.

In Fig. 2, we show the behavior of these two contributions for a cell of type $(+)$. These results were obtained under very general reasoning, and thus they are expected to hold qualitatively for different models of interactions as 
long as the dynamics inside each cell is sufficiently mixed and the phase-space volume is preserved [13].

We have verified that this qualitative behavior is the same for different sizes of the disc, the only change being the values for the saturation plateaus of the geometric contribution [Fig. 2(a)] and the slope of the linear dependence of the dynamical contribution on the local gradients [Fig. 2(b)]. In particular, we have found (not shown) that the variation, from the lower to the upper plateau, of $\alpha_{G}$ is much smaller ( $\sim 20$ times) for the $(-)$ cell than for the $(+)$ cell and similar for $\gamma_{G}$. Therefore, these quantitative values characterize the geometry of the cell and its dynamical response to the presence of thermodynamic gradients.

Combining the two cells. - We now apply these formulas for the two-cell case: For the (-) cell, the particle currents are $j_{>}$and $j_{L}$, while for the $(+)$, they are $j_{R}$ and $j_{<}$. From our findings for a single cell, we find, to a very good approximation,

$$
\begin{gathered}
\varepsilon_{L}^{-}=-\varepsilon_{R}^{-}=\mathcal{A}^{-} \frac{j_{>}-j_{L}}{j_{>}+j_{L}}, \\
\varepsilon_{L}^{+}=-\varepsilon_{R}^{+}=\mathcal{A}^{+} \frac{j_{R}-j_{<}}{j_{R}+j_{<}}, \\
\nu_{L}^{-}=-\nu_{R}^{-}=\mathcal{B}^{-} \frac{j_{>}-j_{L}}{j_{>}+j_{L}}, \\
\nu_{L}^{+}=-\nu_{R}^{+}=\mathcal{B}^{+} \frac{j_{R}-j_{<}}{j_{R}+j_{<}} .
\end{gathered}
$$

Here and below \pm refers to one of the two cells. Substituting (5) into (2) we obtain the currents $j_{R}$ at the center of the cell in terms of the parameters that define the geometry of the cells as

$$
\begin{aligned}
& j_{L}=\frac{\varrho_{\alpha}^{+}\left(1-\varrho_{\alpha}^{-}\right) j_{>}+\left(1-\varrho_{\alpha}^{+}\right) j_{<}}{1-\varrho_{\alpha}^{-} \varrho_{\alpha}^{+}}, \\
& j_{R}=\frac{\left(1-\varrho_{\alpha}^{-}\right) j_{>}+\varrho_{\alpha}^{-}\left(1-\varrho_{\alpha}^{+}\right) j_{<}}{1-\varrho_{\alpha}^{-} \varrho_{\alpha}^{+}},
\end{aligned}
$$
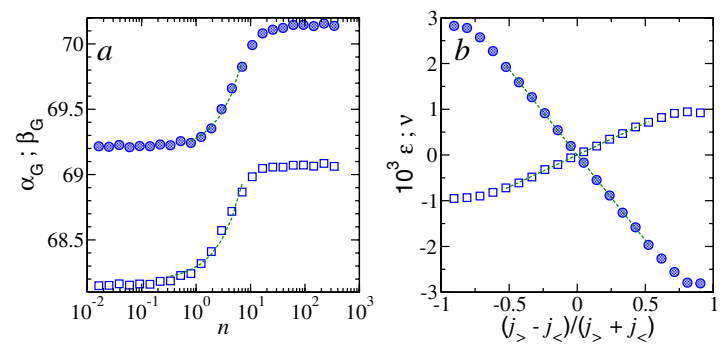

FIG. 2 (color online). (a) Geometrical reflections $\alpha_{G}(n)$ and $\gamma_{G}(n)$ for a single cell with the same dimensions as cell $(+)$, maintained at equilibrium. (b) Dynamical reflections $\varepsilon_{L}$ and $\nu_{L}$ as a function of the local gradients of particle injection rates for the cell $(+)$. Note that by definition $\alpha_{G}=\left(\alpha_{L}+\alpha_{R}\right) / 2$ and similar for $\gamma_{G}$. In both panels, the symbols correspond to the energy reflection (hashed circles) and the particle reflection (open squares) and the dashed lines correspond to linear fits. with effective reflection coefficients given by $\varrho_{\alpha}^{ \pm}=$ $\alpha_{G}\left(n^{ \pm}\right)-\mathcal{A}^{ \pm}$and with $n^{ \pm}$the mean density in the respective cell. One finds finally for the particle current (to within our approximation)

$$
\Phi_{n}=-\frac{\left(1-\varrho_{\alpha}^{-}\right)\left(1-\varrho_{\alpha}^{+}\right)}{1-\varrho_{\alpha}^{-} \varrho_{\alpha}^{+}}\left(j_{<}-j_{>}\right) .
$$

Thus, near equilibrium, $\Phi_{n}$ does not depend on the energy field $q$. In contrast, from (6) it is clear that the current of energy depends on both fields. Substituting (7) into ((6) we find for the dynamical reflections

$$
\nu_{L}=\frac{\mathcal{B}^{-}}{1+\frac{2\left(1-\varrho_{\alpha}^{-} \varrho_{\alpha}^{+}\right) j_{>}}{\left(1-\varrho_{\alpha}^{+}\right)\left(j_{<}-j_{>}\right)}}, \quad \nu_{R}=\frac{\mathcal{B}^{+}}{-1+\frac{2\left(1-\varrho_{\alpha}^{-} Q_{\alpha}^{+}\right) j_{<}}{\left(1-\varrho_{\alpha}^{-}\right)\left(j_{<}-j_{>}\right)}} .
$$

Finally, inserting (6) and (9) in (3), one obtains an expression for the energy current exclusively in terms of the parameters $\Phi_{u}=\Phi_{u}\left(j_{>}, q_{>}, j_{<}, q_{<} ; \varrho_{\alpha}^{ \pm}, \gamma_{G}^{ \pm}, \mathcal{B}^{ \pm}\right)$.

Inverting the imposed fields. - From now on we refer to the case in which the injections at the left are $\left(j_{>}, q_{>}\right)$and at the right $\left(j_{<}, q_{<}\right)$as the positive gradient (PG) and to the inverted case as the negative gradient (NG). Because of the effective interaction among particles the local fields in the cells for the NG are different from those of the PG. Intuitively, this is because the temperature at the junction of the two cells changes when one goes from PG to NG. In particular, the energy reflection coefficients in (9) are not invariant with respect to the sign of the gradient. Let $n^{ \pm}$be the particle density for PG and let $\tilde{n}^{ \pm}$be the particle densities for the NG. Defining $\tilde{\varrho}_{\alpha}^{ \pm}=\alpha_{G}\left(\tilde{n}^{ \pm}\right)-\mathcal{A}^{ \pm}$and using the tilde quantities in (9), one obtains in analogy with (3) for the NG

$$
\tilde{\Phi}_{u}=\frac{\tilde{\tau}_{L}^{\gamma} q_{<}-\tilde{\tau}_{R}^{\gamma} q_{>}}{1-\tilde{\gamma}_{L}^{+} \tilde{\gamma}_{R}^{-}},
$$

with $\tilde{\tau}_{L}^{\gamma}=\left(1-\tilde{\gamma}_{L}^{-}\right)\left(1-\tilde{\gamma}_{L, R}^{+}\right)$and $\tilde{\gamma}_{L}^{ \pm}=\tilde{\gamma}_{G}^{ \pm} \pm \tilde{\nu}^{ \pm}$.

To quantify the TR we define the thermal rectification index as the quotient between the heat currents for the PG and NG,

$$
\Theta_{u}=\frac{\max \left\{\left|\Phi_{u}\right|,\left|\tilde{\Phi}_{u}\right|\right\}}{\min \left\{\left|\Phi_{u}\right|,\left|\tilde{\Phi}_{u}\right|\right\}},
$$

so that $\Theta_{u} \geq 1$. For zero rectification $\Theta_{u}=1$.

Results. - In Fig. 3, we show in a density plot the logarithm of the thermal rectification index $\Theta_{u}$ as a function of the external gradient. A large TR is found in a region where $\delta j$ and $\delta q$ are linearly related. The maximal value of $\Theta_{u}$ observed in Fig. 3 corresponds to heat currents $\Phi_{u}$ and $\tilde{\Phi}_{u}$ where one is 3 orders of magnitude smaller than the other.

As found in previous models, the magnitude of TR depends on the external gradients. For our type of models the largest values of $\Theta_{u}$ are obtained when either $\Phi_{u}$ or $\tilde{\Phi} q_{u}$ (but no both) is very small. This means that TR is maximal when for a given external gradient the system behaves as an insulator. If for a given gradient $(\delta j, \delta q)$ 


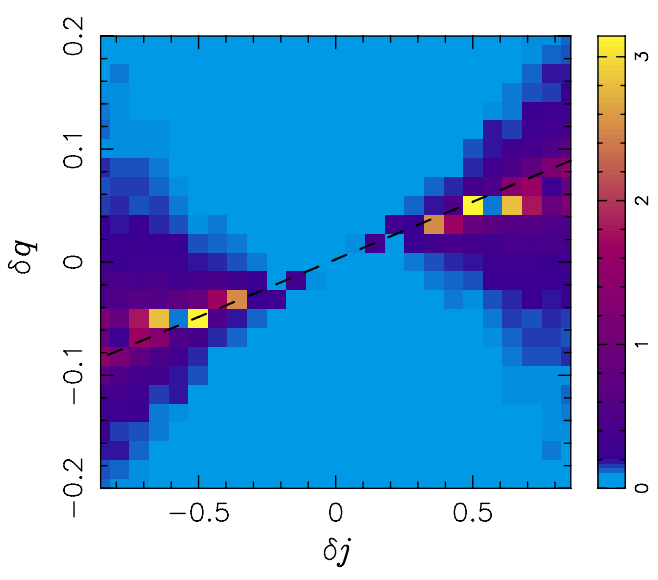

FIG. 3 (color online). Color density plot of the logarithm of the thermal rectification index $\log \left(\Theta_{u}\right)$ as a function of the external gradients $\delta j$ and $\delta q$ for the billiard described in Fig. 1. By construction the data are symmetric along the diagonal. The dashed line corresponds to a fit to the numerical data of the zerocurrent condition (10).

$\Phi_{u}=0$, then for the inverted gradient $(-\delta j,-\delta q) \tilde{\Phi}_{u}$ will not in general be zero. This is because the reflection probabilities will be different through their dependence on the local thermodynamic fields.

Equating $\Phi_{u}$ to zero we obtain a condition that involves the reflection probabilities $\gamma$ :

$$
\frac{\left(1-\gamma_{L}^{-}\right)\left(1-\gamma_{L}^{+}\right)}{\left(1-\gamma_{R}^{-}\right)\left(1-\gamma_{R}^{+}\right)}=\frac{q_{<}}{q_{>}} .
$$

This equation states the values of the external gradient for which our model behaves as a heat insulator. The dashed line in Fig. 3 corresponds to a linear fit to the condition (10) computed from the values obtained from the numerical simulation.

Discussion and conclusions. - In our model TR is due to the dynamical response of each cell to a given external gradient and thus to the interacting character of the particles. If both cells and their discs are identical, then the response for the PG is identical to the one for the NG and there is no TR. On the other hand, it is tempting to consider the opposite case when the domains are equal but the cell (-) has no disc at all. While we have observed TR for this case, we do not report further on this as our analytical treatment is not longer valid.

As we have seen before, rectification has a geometric component (occurring when $\delta j=0$ ). For the billiard of Fig. 1 a thermal rectification of $\Delta_{u} \approx 1.04$ is obtained when the geometric component for the PG and the NG takes values in the lower and upper plateaus, respectively.

This rectification power can be enhanced to much larger values when the billiard behaves as an insulator for positive or negative gradient, and this can be achieved when $\delta j \neq 0$.

The geometric component also allows a rectification of the particle current. From (8), it is clear that the particle current $\Phi_{n}$ is zero only when $\delta j=0$. Therefore, this rectification cannot be enhanced.

For open billiards like the one studied here, the finite particle current contributes to the TR only through the dynamical component $\nu^{ \pm}$. In practice, this situation corresponds to the transport in porous materials where the energy is carried by the particles. However, for the analogous closed billiard for which only energy is exchange between the system and the reservoirs large thermal rectification have been numerically observed.

In this Letter, we have established the possibility to observe thermal rectification in billiardlike mechanical systems. When the particles inside the billiard interact, the response of the system to an external thermodynamic gradient is dynamically controlled. We have studied this dynamical effect in terms of the particle and energy reflection coefficients for which an analytical phenomenological formulation in terms of the local thermodynamic gradients is possible. This dynamical response leads to the possibility that the system behaves as an insulator for one gradient and as a conductor with the inverted gradient, in which case large thermal rectification is obtained. Our analytical treatment is independent of the specifics and generalizes to many other effective interactions. However, a dependence on the local fields induced by any generic effective manybody interaction is likely. This leads us to conjecture that TR could be observable in mesoscopic asymmetric billiard systems for which the gas of electrons is not free.

The authors acknowledge fruitful discussions with Tomaž Prosen and support by the Fonds National Suisse. C. M.-M. thanks the CIC in Cuernavaca, Mexico, where part of this work was done.

[1] F. Bonetto, J. L. Lebowitz, and L. Rey-Bellet, in Mathematical Physics 2000 (Imperial College Press, London, 2000), p. 128.

[2] S. Lepri, R. Livi, and A. Politi, Phys. Rep. 377, 1 (2003).

[3] D. G. Cahill et al., J. Appl. Phys. 93, 793 (2003).

[4] P. W. O'Callaghan, S. D. Probert, and A. Jones, J. Phys. D 3, 1352 (1970).

[5] M. Terraneo, M. Peyrard, and G. Casati, Phys. Rev. Lett. 88, 094302 (2002).

[6] B. Li, L. Wang, and G. Casati, Phys. Rev. Lett. 93, 184301 (2004).

[7] B. Li, L. Wang, and G. Casati, Appl. Phys. Lett. 88, 143501 (2006).

[8] B. Li, J.-H. Lan, and L. Wang, Phys. Rev. Lett. 95, 104302 (2005).

[9] D. Segal and A. Nitzan, Phys. Rev. Lett. 94, 034301 (2005).

[10] C. Mejía-Monasterio, H. Larralde, and F. Leyvraz, Phys. Rev. Lett. 86, 5417 (2001).

[11] H. Larralde, F. Leyvraz, and C. Mejía-Monasterio, J. Stat. Phys. 113, 197 (2003).

[12] J.-P. Eckmann and L.-S. Young, Commun. Math. Phys. 262, 237 (2006).

[13] J.-P. Eckmann, C. Mejía-Monasterio, and E. Zabey, condmat/0510698 [J. Stat. Phys. (to be published)]. 